\title{
The Interplay of Primary Tumor Location and KRAS Mutation Status in Patients with Synchronous Colorectal Cancer Liver Metastases: Current Data and Unanswered Questions
}

\author{
Georgios Antonios Margonis, MD, PhD ${ }^{1,3}$, Nikolaos Andreatos, $\mathrm{MD}^{2}$, Martin E. Kreis, $\mathrm{MD}^{3}$, and \\ Michael D'Angelica, MD ${ }^{1}$ \\ ${ }^{1}$ Department of Surgery, Memorial Sloan Kettering Cancer Center, New York, NY; ${ }^{2}$ Department of Internal Medicine and \\ Taussig Cancer Institute, Cleveland Clinic, Cleveland, OH; ${ }^{3}$ Department of Surgery, Campus Benjamin Franklin, Charité, \\ University Medicine, Berlin, Germany
}

Primary tumor location (PTL) and KRAS mutation status are gradually gaining acceptance as useful surrogates of tumor biology and have an evolving role in the prognostication of patients who undergo hepatectomy for colorectal cancer liver metastases (CRLM). Although the association of each of these factors with prognosis has been repeatedly assessed, data on their interplay are far more limited, rendering the present work pertinent. ${ }^{1}$ The authors first analyzed survival outcomes according to PTL [rightsided (RS) vs. left-sided (LS)] and KRAS mutation status [mutant KRAS (mutKRAS) vs. wild-type KRAS (wtKRAS)] among 227 patients who underwent resection for synchronous CRLM between 2006 and 2015 at the Severance Hospital in Seoul; subsequently, the outcomes of patients belonging to the 4 subgroups defined by the combination of PTL and KRAS mutation status (namely RS/mutKRAS, RS/wtKRAS, LS/mutKRAS and LS/ wtKRAS) were independently considered. ${ }^{1}$ To our knowledge, this is the first study to examine the interplay of these factors in patients with synchronous CRLM who are traditionally thought to exhibit a more aggressive disease course compared to patients with metachronous tumors. ${ }^{2}$ However, given the lack of definitive proof that

(C) Society of Surgical Oncology 2020

First Received: 31 August 2020

Accepted: 3 September 2020;

Published Online: 21 September 2020

G. A. Margonis, $\mathrm{MD}, \mathrm{PhD}$

e-mail: antonis.margonis@gmail.com synchronous and metachronous disease are really distinct entities, it would perhaps have been more interesting to assess both of these subgroups simultaneously.

The study confirmed that patients with RS primary tumors experience worse outcomes, a finding in line with prior reports and a recent comprehensive meta-analysis; nonetheless, these results may be partly dependent on length of follow-up as the prognostic effect of primary tumor location has been shown to diminish over time. ${ }^{3,4}$ On the other hand, while trends suggesting that the presence of KRAS mutations may be associated with inferior prognosis were noted, they failed to reach statistical significance. As the statistical power of the study was significantly limited and the effect of KRAS mutation status on outcomes is now thought to be rather modest, these results are not surprising. ${ }^{5}$ In fact, a number of reports similarly failed to confirm the prognostic impact of KRAS mutation status, igniting a controversy that was only resolved via larger, more adequately powered studies and pooled analyses. ${ }^{6-8}$ While KRAS mutation status failed to demonstrate a statistically significant association with prognosis even when examined separately among patients with RS and LS primaries, the RS/mutKRAS subgroup demonstrated very poor outcomes. The lack of reported interactions between these two variables, if not a byproduct of limited statistical power, suggests that these results may stem from the superimposition of two distinct determinants of poor prognosis (albeit KRAS status contributed a trend rather than a statistically significant association), rather than a PTL-specific effect of KRAS mutations. The relatively limited study population, especially with respect to patients with mutKRAS and wtKRAS RS tumors $(n=20$ and 21, 
respectively) renders the precise size of this combined prognostic effect unclear; specifically, the hazard ratio for overall survival (OS) associated with the presence of a RS/mutKRAS primary tumor ranged from 1.187 to 5.361 . Larger studies are clearly required to refine this estimate and guide the development of future prognostic models.

The possible interplay of PTL and KRAS mutation status has been previously assessed in "all-comers" with surgically resectable CRLM. In 2016, Sasaki et al. reported that the presence of KRAS mutations was prognostic of inferior recurrence-free survival (RFS) and OS only among patients with LS primaries, in a single-institution cohort of 426 patients. ${ }^{9}$ While the authors did not perform formal statistical comparisons of the survival of patients with RS/ wtKRAS versus LS/wtKRAS and RS/mutKRAS versus LS/mutKRAS tumors, there was a considerable numerical difference in the former (median OS: 65.8 vs. 56.4 months, respectively) while patients with RS/mutKRAS versus LS/mutKRAS fared similarly (median OS: 46.8 vs. 44.0 months, respectively). In turn, this may have served to increase the prognostic differential between LS/wtKRAS and LS/mutKRAS tumors while minimizing that between RS/wtKRAS and RS/mutKRAS tumors, which could help explain the primary findings of the study. A subsequent report by Gofreddo et al. that relied on a cohort of 2655 patients from the National Cancer Database approached the question from a different angle; after demonstrating that both PTL and KRAS mutation status were independent predictors of OS, the authors showed that the presence of a RS primary was associated with worse outcomes among patients with both mutKRAS and wtKRAS tumors. ${ }^{10}$ Even though the authors noted that the survival difference between patients with RS and LS tumors was less pronounced in the mutKRAS cohort, patients with RS/mutKRAS tumors ultimately fared worse, in line with the current work. Similarly, Margonis et al. recently demonstrated that while patients with RS/mutKRAS tumors fared only slightly worse in absolute terms with respect to 5-year OS compared with patients with LS/mutKRAS tumors $(32.8 \%$ vs. $34.0 \%$, respectively a difference that was not statistically significant), the respective difference was far more pronounced for patients with RS/wtKRAS versus LS/wtKRAS tumors $(43.7 \%$ vs. $55.5 \%, p=0.02) .{ }^{11}$ While the specific estimates vary, the broad picture that emerges from these combined results and the present report is that both RS primary tumors and the presence of KRAS mutations are likely predictors of poor survival, with the combined effect of the two factors leading to especially unfavorable outcomes. While the latter is primarily driven by PTL in the present study, accurate assessment of the respective prognostic effects of these factors can only be obtained via analysis of much larger patient cohorts.
The prognostic importance of PTL (and its possible predictive role in the selection of first-line biologics) is becoming increasingly clear, but the molecular aberrations that render RS and LS tumors so notably distinct have yet to be completely elucidated. ${ }^{12}$ As the data above suggest, while KRAS mutations have been reported to be more frequent in RS tumors, they do not alone explain the observed prognostic disparities which extend to patients with wtKRAS tumors. ${ }^{11}$ Instead, the frequent presence of mutations in other important genetic loci (e.g., BRAF) among RS/wtKRAS tumors is likely a major contributor to their unfavorable biologic behavior. ${ }^{13,14}$ On the other hand, the even distribution (between RS and LS tumors) of the SMAD4 and p53 mutations, which when combined with KRAS mutations confer especially adverse outcomes might explain why RS and LS mutKRAS patients fare comparably. ${ }^{15,16}$ In fact, KRAS mutation is not prognostic unless there is a coexisting TP53 mutation. ${ }^{15,16}$

While the identification of patient subgroups with distinct prognosis is an important first step before changes in management can be contemplated, the authors' suggestion that patients with RS/mutKRAS tumors may not be good candidates for upfront surgery should not be interpreted as a clinical conclusion stemming from the data but as an invitation for further research. Nonetheless, the authors indirectly raise an important point as to the optimal sequencing of surgery and systemic therapy in CRLM, an area of practice which is neither standardized nor supported by high-quality evidence. The evolution of prognostic modeling in CRLM, which recently incorporated KRAS mutation status (e.g., GAME and modified CRS scores) and can in the future also include PTL will help to improve preoperative risk stratification and, ultimately, facilitate patient allocation in clinical trials that can definitively identify optimal treatment sequencing strategies. ${ }^{17,18}$ Moreover, the collective clinical experience of multidisciplinary tumor boards at specialized centers is and will likely remain invaluable, given the considerable complexity that characterizes the management of patients with CRLM.

Last but not least, the authors' decision to differentiate between LS colon and rectal tumors is methodologically and biologically sound; indeed, emerging evidence suggests that rectal tumors have a distinct molecular profile, which differs substantially from that of other LS tumors thus undermining any justification for treating them as similar entities for the purpose of analysis. ${ }^{14}$ While the main findings of the study were not substantially altered by excluding patients with rectal tumors and the subanalysis assessing outcomes of patients with mutKRAS versus wtKRAS status in that group yielded similar results with that of the unselected LS cohort, relatively low power limited the ability to identify underlying differences. 
Interestingly, a recent report by Amini et al. demonstrated that mutKRAS status was independently associated with worse outcomes in patients with CRLM arising from colon, but not rectal cancer. ${ }^{19}$ While in the study by Kim et al., patients with mutKRAS rectal tumors fared somewhat worse than those with wtKRAS tumors in absolute terms and inability to demonstrate a significant difference in a study does not conclusively disprove its existence, these results nonetheless suggest that KRAS mutations may have distinct implications for patients with rectal tumors. Further studies in much larger cohorts will be helpful in elucidating the prognostic impact of KRAS and other candidate biomarkers in this patient group.

In conclusion, Kim et al. have presented interesting albeit inconclusive data on the open question of the interplay between tumor location and KRAS mutation status and emphasize the prognostic importance of the former while simultaneously bringing attention to the somewhat arbitrary nature of the right versus left dichotomy. Importantly, they have set the stage for similar analyses of larger cohorts, preferably, including information on additional molecular characteristics, such as microsatellite instability (MSI) status and other somatic mutations, that may definitively answer these highly interesting questions.

DISCLOSURES The authors declare no conflicts of interest.

\section{REFERENCES}

1. Kim HS, Lee, JM, Kim, HS, et al. Prognosis of synchronous colorectal liver metastases after simultaneous curative-intent surgery according to primary tumor location and KRAS mutational status. Ann Surg Oncol. 2020. https://doi.org/10.1245/ s10434-020-09041-0.

2. Adam R, de Gramont A, Figueras J, et al. Managing synchronous liver metastases from colorectal cancer: a multidisciplinary international consensus. Cancer Treat Rev. 2015;41:729-41.

3. Wang XY, Zhang R, Wang Z, et al. Meta-analysis of the association between primary tumour location and prognosis after surgical resection of colorectal liver metastases. Br J Surg. 2019;106:1747-60.

4. Creasy JM, Sadot E, Koerkamp BG, et al. The impact of primary tumor location on long-term survival in patients undergoing hepatic resection for metastatic colon cancer. Ann Surg Oncol. 2018;25:431-8.

5. Margonis GA, Buettner S, Andreatos N, et al. Prognostic factors change over time after hepatectomy for colorectal liver metastases: a multi-institutional, international analysis of 1099 patients. Ann Surg. 2019;269:1129-37.
6. Petrowsky H, Sturm I, Graubitz O, et al. Relevance of Ki-67 antigen expression and K-ras mutation in colorectal liver metastases. Eur J Surg Oncol. 2001;27:80-7.

7. Margonis GA, Spolverato G, Kim Y, Karagkounis G, Choti MA, Pawlik TM. Effect of KRAS mutation on long-term outcomes of patients undergoing hepatic resection for colorectal liver metastases. Ann Surg Oncol. 2015;22:4158-65.

8. Brudvik KW, Kopetz SE, Li L, Conrad C, Aloia TA, Vauthey JN. Meta-analysis of KRAS mutations and survival after resection of colorectal liver metastases. Br J Surg. 2015;102:1175-83.

9. Sasaki K, Margonis GA, Wilson A, et al. Prognostic implication of kras status after hepatectomy for colorectal liver metastases varies according to primary colorectal tumor location. Ann Surg Oncol. 2016;23:3736-43.

10. Goffredo P, Utria AF, Beck AC, et al. The prognostic impact of KRAS mutation in patients having curative resection of synchronous colorectal liver metastases. J Gastrointest Surg. 2019;23:1957-63.

11. Margonis GA, Amini N, Buettner S, et al. The prognostic impact of primary tumor site differs according to the KRAS mutational status: a study by the international genetic consortium for colorectal liver metastasis. Ann Surg. 2019.

12. Holch JW, Ricard I, Stintzing S, Modest DP, Heinemann V. The relevance of primary tumour location in patients with metastatic colorectal cancer: a meta-analysis of first-line clinical trials. Eur J Cancer. 2017;70:87-98.

13. Gagniere J, Dupre A, Gholami SS, et al. Is hepatectomy justified for BRAF mutant colorectal liver metastases? A multi-institutional analysis of 1497 patients. Ann Surg. 2020;271:147-54.

14. Loree JM, Pereira AAL, Lam M, et al. Classifying colorectal cancer by tumor location rather than sidedness highlights a continuum in mutation profiles and consensus molecular subtypes. Clin Cancer Res. 2018;24:1062-72.

15. Kawaguchi Y, Kopetz S, Newhook TE, et al. Mutation status of RAS, TP53, and SMAD4 is superior to mutation status of RAS alone for predicting prognosis after resection of colorectal liver metastases. Clin Cancer Res. 2019;25:5843-51.

16. Datta J, Smith JJ, Chatila WK, et al. Coaltered Ras/B-raf and TP53 is associated with extremes of survivorship and distinct patterns of metastasis in patients with metastatic colorectal cancer. Clin Cancer Res. 2020;26:1077-85.

17. Margonis GA, Sasaki K, Gholami S, et al. Genetic and Morphological Evaluation (GAME) score for patients with colorectal liver metastases. Br J Surg. 2018;105:1210-20.

18. Brudvik KW, Jones RP, Giuliante F, et al. RAS mutation clinical risk score to predict survival after resection of colorectal liver metastases. Ann Surg. 2019;269:120-6.

19. Amini N, Margonis GA, Kreis ME, et al. Prognostic impact of KRAS mutational status in patients with colorectal cancer liver metastases differs according to the location of the primary tumor. J Am Coll Surg. 2019;229:S69-70.

Publisher's Note Springer Nature remains neutral with regard to jurisdictional claims in published maps and institutional affiliations. 